UDC: $517.938: 517.552]: 539.37$

44 (LXX) 1 (2020), 53-60

DOI: $10.37560 /$ matbil2010053s

\title{
COMPARISON OF TWO NUMERICAL METHODS FOR FRACTIONAL-ORDER RÖSSLER SYSTEM
}

\author{
YLLDRITA SEFERI ${ }^{1}$, GJORGJI MARKOSKI ${ }^{2}$, AND ALEKSANDAR GJURCHINOVSKI ${ }^{3}$
}

\begin{abstract}
In this paper, we numerically study the chaotic behavior of the fractional-order Rössler system comparing the numerical solutions of the system with Adams-Bashforth-Moulton method (FABM) and Fractional Multistep Differential Transformation method (FMDTM). The fractional derivatives are described in the Caputo sense. FABM method acts like a predictorcorrector pair compared with FMDTM, which is a semi-numerical method that exploits the power-series representation of the solution. Numerically obtained results are analyzed to compare the different integration algorithms. We quantify the distinction between the methods for arbitrary chosen system parameters in the chaotic regime. We have shown numerically that the difference between the results is less pronounced as the value of the fractional-order becomes closer to one.
\end{abstract}

\section{INTRODUCTION}

The theory of fractional-order derivatives (or derivatives of non-integer order) goes back to Leibniz's letter to L'Hospital, dated 30 September 1695, in which the meaning of derivative of order one-half was discussed. Although fractional calculus has a 300-year-old history, its applications to physics and engineering are just recent focus of interest.

In this paper, the approximate solutions of the well-known fractional-order Rössler system are investigated using different numerical methods. For this system chaotic behavior exists for fractional-order derivatives as low as 2.4 [8], but in our case-study, we explore the interval $(0,1]$. Like many other studies of fractional-order dynamics, we investigate the behavior of Rössler system through numerical simulations, in this case by comparing fractional Adams-BashforthMoulton method (FABM) and fractional Multistep Differential Transformation method (FMDTM) [2, 3]. 


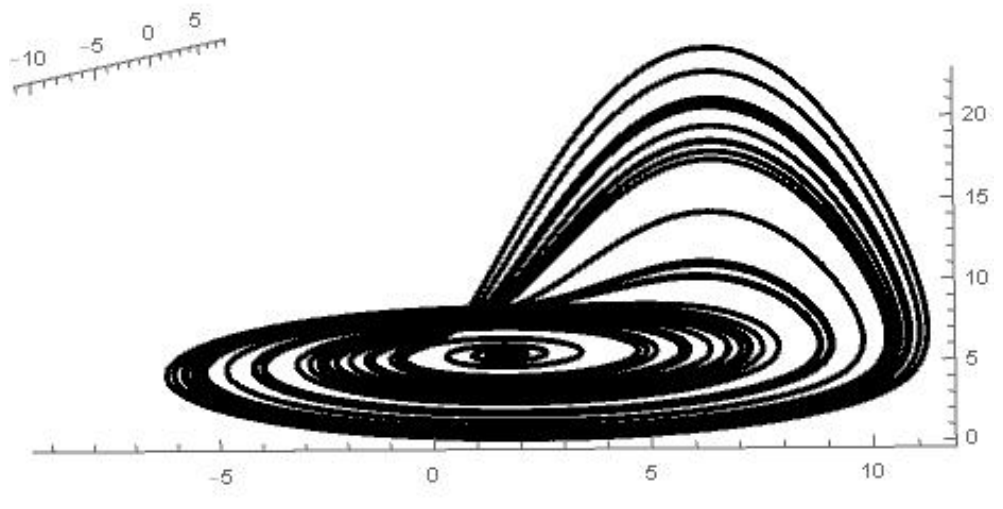

FIgURE 1. Simulation result of the fractional-order Rossler system (1.1) in state space for parameters $a=0.2, b=0.2, c=5.7$ and fractional order $v=0.9$ for simulation time $120 \mathrm{~s}$, with initial conditions $(x(0), y(0), z(0))=(0,0,0)$.

In the following we will explore the numerical solution of the fractional Rössler system by fixing the parameter values at $a=0.1, b=0.1$ and $c=14$.

In 1976 Rössler [5] proposed a simple nonlinear system with a strange attractor with a single quadratic nonlinearity in the differential equations, with no a-priori relevance to any real physical system at that time. The original theoretical equations were later found to be useful in modeling equilibrium chemical reactions. This attractor can have only one chaotic manifold, which is a solution to the following equations for certain parameter values $a, b, c$, and $v$ :

$$
\begin{aligned}
& D_{t}^{v} x(t)=-(y(t)+z(t)) \\
& D_{t}^{v} z(t)=x(t)+a y(t) \\
& D_{t}^{v} y(t)=b+x(t) z(t)-c z(t) .
\end{aligned}
$$

Here, $D_{t}^{v}$ is time fractional-order derivative defined in the Caputo sense. For example, for $a=0.2, b=0.2, c=5.7$, and fractional order $v=0.9$ there exist a chaotic scroll attractor (see Fig. 1) and an unstable stationary point at

$$
x_{0}=\left(\frac{c-\sqrt{c^{2}-4 a b}}{2},-\frac{c-\sqrt{c^{2}-4 a b}}{2 a}, \frac{c-\sqrt{c^{2}-4 a b}}{2 a}\right) .
$$

2000 Mathematics Subject Classification. 34C28, 34A08, 74H15.

Key words and phrases. Fractional-order Rössler system, Adams-Bashforth-Moulton method, Fractional Multistep Differential Transformation method, fractional dynamical system, numerical analysis. 


\section{Fractional Multi-step differential transform Method}

The Discrete Transformation Method (DTM) is used to provide approximate solutions for a wide class of nonlinear problems in terms of convergent series with easily computable components. The method, however, has some drawbacks: the series solution always converges in a very small region and it has slow convergent rate in the wider region [2]. To overcome this shortcoming, we present in this section the FMDTM that was originally developed for the numerical solutions of ordinary differential equations [6]. For this purpose, we consider the following nonlinear initial value problem:

$$
f\left(t, x, x^{\prime}, \ldots, x^{(v)}\right)=0
$$

subject to initial conditions $x^{(k)}(0)=c_{k}, \quad k=0,1, \ldots, v-1$.

Let $[0, T]$ be the interval over which we want to find the solution of the initial value problem (2.1). In actual applications of the DTM, the approximate solution of the initial value problem 2.1) can be expressed by the finite series,

$$
x(t)=\sum_{n=0}^{N} a_{n} t^{n}, \quad t \in[0, T]
$$

The Multi-step approach introduces a new idea for constructing the approximate solution. Assume that the interval $[0, T]$ is divided into $\mathrm{M}$ subintervals $\left[t_{m-1}, t_{m}\right]$, $m=1,2, \ldots, M$ of equal step size $h=\frac{T}{M}$ by using the nodes $t_{m}=m h$ [6]. The main idea of the FMDTM is in the following. First, we apply the DTM to 2.1 over the interval $\left[0, t_{1}\right]$, we will obtain the following approximate solution:

$$
x_{1}(t)=\sum_{n=0}^{K} a_{1 n} t^{n}, \quad t \in\left[0, t_{1}\right]
$$

using the initial conditions $x_{1}^{(k)}(0)=c_{k}$. For $m \geq 2$ and at each subinterval $\left[t_{m-1}, t_{m}\right]$ we will use the initial conditions $x_{m}^{(k)}\left(t_{m-1}\right)=x_{m-1}^{(k)}\left(t_{m-1}\right)$ and apply the DTM to 2.1 over the interval $\left[t_{m-1}, t_{m}\right]$, where $t_{0}$ in $F(k)=\frac{1}{k !}\left[\frac{d^{k} f(t)}{d t^{k}}\right]_{t=t_{0}}$ is replaced by $t_{m-1}$. The process is repeated and generates a sequence of approximate solutions $x_{m}(t), m=1,2, \ldots, M$, for the solution $x(t)$ :

$$
x_{m}(t)=\sum_{n=0}^{K} a_{m n}\left(t-t_{m-1}\right)^{n}, \quad t \in\left[t_{m}, t_{m+1}\right]
$$

where $N=K M$. In fact the FMDTM assumes the following solution:

$$
x(t)= \begin{cases}x_{1}(t), & t \in\left[0, t_{1}\right] \\ x_{2}(t), & t \in\left[t_{1}, t_{2}\right] \\ \vdots & \\ x_{M}(t), & t \in\left[t_{M-1}, t_{M}\right]\end{cases}
$$


The new algorithm, FMDTM, is simple for computational performance for all values of $h$. It is easily observed that if the step size $h=T$, then the FMDTM reduces to the classical DTM 6, 7, 4,

2.1. FMDTM for Rossler System. Now, we take the system (1.1) into consideration. According to the FMDTM, the series solution for Rössler system is given by:

$$
(x(t), y(t), z(t))=\left\{\begin{array}{l}
\sum_{n=0}^{N}\left(X_{1}(t), Y_{1}(t), Z_{1}(t)\right) t^{n}, t \in\left[0, t_{1}\right] \\
\sum_{n=0}^{N}\left(X_{2}(t), Y_{2}(t), Z_{2}(t)\right)\left(t-t_{1}\right)^{n}, t \in\left[t_{1}, t_{2}\right] \\
\vdots \\
\sum_{n=0}^{N}\left(X_{M}(t), Y_{M}(t), Z_{M}(t)\right)\left(t-t_{M-1}\right)^{n}, t \in\left[t_{M-1}, t_{M}\right]
\end{array}\right.
$$

where $X_{i}(n), Y_{i}(n)$ and $Z_{i}(n)$, for $i=1,2, . ., M$, satisfy the following recurrence relations:

$$
\begin{aligned}
& X_{i}(k+1)=\frac{-1}{(k+1) !}\left(Y_{i}(k)+Z_{i}(k)\right) \\
& Y_{i}(k+1)=\frac{1}{(k+1) !}\left(X_{i}(k)+a Y_{i}(k)\right) \\
& Z_{i}(k+1)=\frac{1}{(k+1) !}\left(\sum_{l=0}^{k} Z_{i}(l) X_{i}(l)-c Z_{i}(k)+b \delta(k)\right)
\end{aligned}
$$

such that $X_{1}(0)=x(0), Y_{1}(0)=y(0), Z_{1}(0)=z(0)$ and $X_{i}(0)=X_{i-1}(0)$, $Y_{i}(0)=Y_{i-1}(0), Z_{i}(0)=Z_{i-1}(0)$, for $i=2,3, \ldots, M$. Numerical results are taken using FMDTM like an analitical method with aim to show how FMDTM will agree with FABM in the numerical approximation of the solutions of the system 6. FABM act like a predictor-corrector pair which represents an amalgamation between fractional Adams Bashforth FAB and fractional Adams Moulton FAM methods [7, 2, 3].

\section{Adams-Bashforth-Moulton Method}

We consider the initial value problem (2.1). We assume a solution of (2.1) on some interval $[0, T]$ to construct approximate solution values $x_{j} \approx x(j)$ at the grid points $t_{j}=j h,(j=0,1,2, \ldots, N)$ for $h=\frac{T}{N}[3$. The initial value problem 2.1] is equivalent to the Volterra integral equation:

$$
x(t)=\sum_{k=0}^{\lceil v\rceil-1} \frac{x_{0}^{(k)}}{k !} t^{k}+\frac{1}{\Gamma(v)} \int_{0}^{t}(t-\tau)^{v-1} f(\tau, x(\tau)) d \tau
$$

The main part of the algorithm for (FAB)

$$
x[j]=\sum_{k=0}^{\lceil v\rceil-1} \frac{(j h)^{k}}{k !} x_{0}^{(k)}+\frac{h^{v}}{\Gamma(v+1)} \sum_{k=0}^{j-1} b[j-k] f(k h, x[k])
$$


where $b[j-k]$ are the weights which depends only on the difference $(j-k)$ because of the convolution structure of $b_{k, j}$.

Using FAB and FAM, the main part of the FABM of the algorithm is:

$$
\begin{aligned}
& p=\sum_{k=0}^{\lceil v\rceil-1} \frac{(j h)^{k}}{k !} x_{0}^{(k)}+\frac{h^{v}}{\Gamma(v+1)} \sum_{k=0}^{j-1} b[j-k] f(k h, x[k]) \\
& x[j]=\sum_{k=0}^{\lceil v\rceil-1} \frac{(j h)^{k}}{k !} x_{0}^{(k)}+\frac{h^{v}}{\Gamma(v+2)}\left(f(j h, p)+\left((j-1)^{v+1}-\right.\right. \\
&\left.\left.-(j-v-1) j^{v}\right)(f(0, x[0]))+\sum_{k=0}^{j-1} a[j-k] f(k h, x[k])\right)
\end{aligned}
$$

The corrector part of 2.2 is denoted by $x[j]$ with weight $a[j-k](3.4)$, where $p$ represents FAB, which in this case acts like a predictor [1, 2, 3].

3.1. FABM for Rossler System. In the case of fractional-order Rössler system (1.1), the method will take the form:

Predictor of the algorithm:

$$
\begin{aligned}
& p[j]=x_{0}+\frac{h^{v}}{\Gamma(v+1)} \sum_{k=0}^{j-1} b[j-k](-(y[k]+z[k])) \\
& q[j]=y_{0}+\frac{h^{v}}{\Gamma(v+1)} \sum_{k=0}^{j-1} b[j-k](x[k]+a y[k]) \\
& \left.r[j]=z_{0}+\frac{h^{v}}{\Gamma(v+1)} \sum_{k=0}^{j-1} b[j-k](b+x[k] z[k]-c z[k])\right)
\end{aligned}
$$

Corrector of the algorithm:

$$
\begin{gathered}
x[j]=x_{0}+\frac{h^{v}}{\Gamma(v+2)}\left(-(q[j]+r[j])+(j-1)^{v+1}-\right. \\
\left.-(j-v-1) j^{v}\right)\left(-(y[0]+z[0])+\sum_{k=1}^{j-1} a[j-k](-(y[k]+z[k]))\right. \\
y[j]=y_{0}+\frac{h^{v}}{\Gamma(v+2)}\left((p[j]+a q[j])+\left((j-1)^{v+1}-\right.\right. \\
\left.\left.-(j-v-1) j^{v}\right)(x[0]+a y[0])+\sum_{k=1}^{j-1} a[j-k](x[k]+a y[k])\right) \\
z[j]=z_{0}+\frac{h^{v}}{\Gamma(v+2)}\left((b+p[j] r[j]-c r[j])+\left((j-1)^{v+1}-(j-v-1) j^{v}\right) .\right.
\end{gathered}
$$




$$
\left.\cdot(b+x[0] z[0]-c z[0])+\sum_{k=1}^{j-1} a[j-k](b+x[k] z[k]-c z[k])\right)
$$

\section{Numerical RESUlts}

The parameters of the fractional-order Rossler system are taken arbitrary, here at particular values $a=0.1, b=0.1$, and $c=14$ (we note that the conclusions in the further analysis are quite general and valid for other parameter values also). We aim to show how FMDTM and FABM methods will agree with each other in the numerical approximation of the solutions of the system. We take the same initial conditions $x(0)=0, y(0)=0$ and $z(0)=0$ in all the simulations. We consider four different values of the fractional-orders within the interval $(0,1]$, that is $v=0.6, v=0.8, v=0.9$ and $v=0.98$.

a

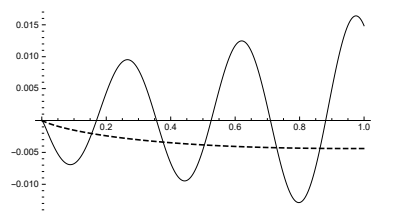

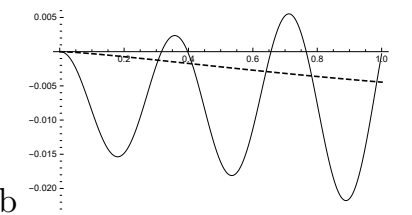

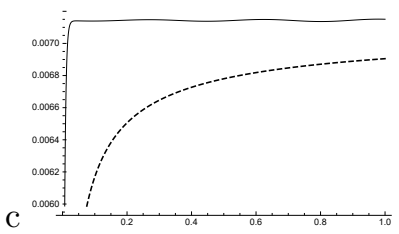

Figure 2. Time-series of system 1.1 with $v=0.6$, initial conditions $(x(0), y(0), z(0))=(0,0,0)$, step size $h=0.001$ and $t \in[0,1]$ (FMDTM - solid line, FABM -dashed line]. a) $x(t) ;$ b) $y(t) ;$ c) $z(t)$
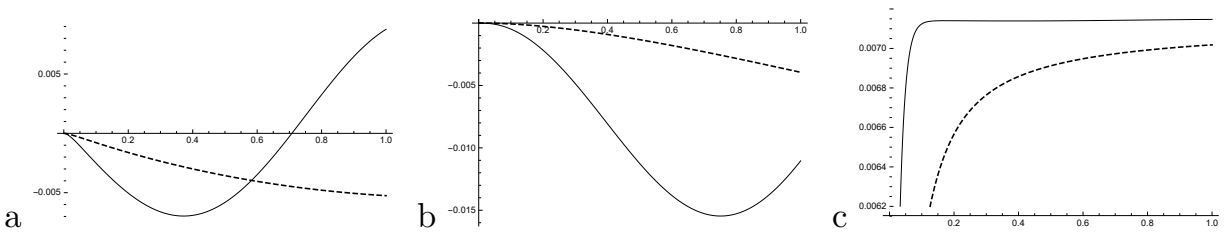

Figure 3. Time-series of system 1.1 with $v=0.8$, initial conditions $(x(0), y(0), z(0))=(0,0,0)$, size is $h=0.001$ and $t \in[0,1]$ (FMDTM solid line, FABM -dashed line]. a) $x(t)$; b) $y(t) ;$ c) $z(t)$

\section{Conclusions}

In this paper, two different numerical approximation schemes (FMDTM and FABM) have been applied to find the time-series solutions of the fractional-order Rössler system. The Wolfram Mathemaica 11 software, was used to obtain and approximate the results shown in this paper. We have aimed to quantify the distinction between the integration methods by depicting the time series for the same system parameters and initial conditions, by varying the order of the fractional 

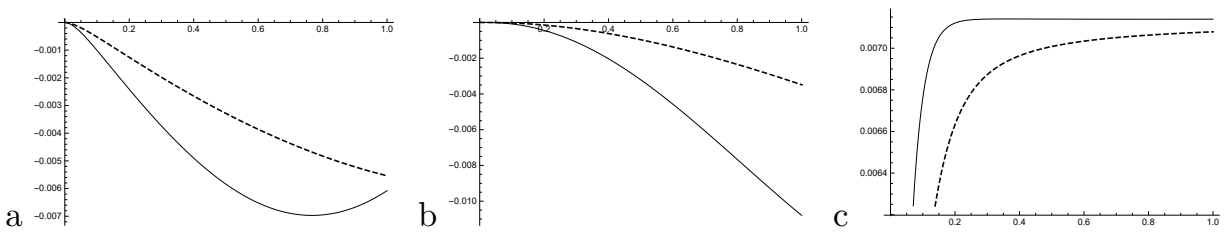

FigurE 4. Time-series of system 1.1 with $v=0.9$, initial conditions $(x(0), y(0), z(0))=(0,0,0)$, size is $h=0.001$ and $t \in[0,1]$ (FMDTM solid line, FABM -dashed line]. a) $x(t) ;$ b) $y(t) ;$ c) $z(t)$
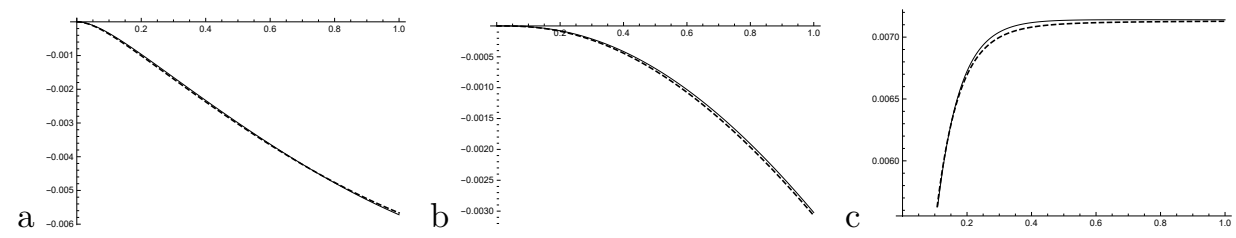

Figure 5. Time-series of system 1.1, whith $v=0.98$, initial conditions $(x(0), y(0), z(0))=(0,0,0)$, step size is $h=0.001$ and $t \in[0,1]$ (FMDTM - solid line, FABM -dashed line]. a) $x(t) ;$ b) $y(t) ;$ c) $z(t)$

derivative $v$. The results show that FABM method generally differ from the time series approximations obtained by FMDTM, except when $v=0.98$.

We have shown numerically that this difference is less pronounced as the value of the fractional-order becomes closer to one. The methods of numerical integration are substantially different with respect to the speed of the numerical computation, since FMDTM is using the time-series values of the previous step as an input for computing the next values, whilst FABM requires a knowledge of the whole history to compute the next step, and thus is much time-consuming with respect to FMDTM.

\section{REFERENCES}

[1] C. Bervillier, Status of the differential transformation method, Applied Mathematics and Computation, 20, (2012), 10158-10170.

[2] L. Changpin, Z. Fanhai, Numerical Methods for Fracional Calculus, (2015), CRC Press Taylor and Francis Group, New York.

[3] K. Diethelm, N. J. Ford and A. D. Freed, A Predictor-Corrector Approach for the Numerical Solution of Fractional Differential Equations, Nonlinear Dynamics, 29, (2001), 3-22.

[4] S. V. Ertürk, Sh. Momani, Solving systems of fractional differential equations using differential transform method, Journal of Computational and Applied Mathematics, 215, (2008), $142-151$.

[5] N.V. Kuznetsov, T.N. Mokaev, P.A. Vasilyev, Numerical justification of Leonov conjecture on Lyapunov dimension of Rossler attractor, Communications in Nonlinear Science and Numerical Simulation, 19, (2013), 1027-1034. 
[6] Z. Odibat, C. Bertelle, M. Aziz-Alaoui, G.H.E. Duchamp, A multi-step differential transform method and application to non-chaotic or chaotic systems, Computer and mathematics with applications, 59, (2010), 1462-1472.

[7] Z. Odibat, Sh. Momani, V.S. Ertürk, Generalized Differential Transform Method: Application to Differential Equations of Fractional Order, Applied Mathematics and Computation, 197, (2008), 467-477.

[8] I. Petras, Fractional-Order Nonlinear Systems Modeling, Analysis and Simulation, (2011), Springer-Verlag Berlin Heidelberg.

1 State University of Tetova,

Ilindenska Nn, 1200 Tetovo, North Macedonia

Email address: ylldrita.seferi@unite.edu.mk

2 Ss. Cyril and Methodius University,

Faculty of Natural Sciences and Mathematics, Institute of Mathematics,

Arhimedova 3, 1000, Skopje, North Macedonia

Email address: gorgim@pmf.ukim.mk

3 Ss. Cyril and Methodius University,

Faculty of Natural Sciences and Mathematics, Institute of Physics,

Arhimedova 3, 1000, Skopje, North Macedonia

Email address: agjurcin@yahoo.com

Received: 29.2 .2020

Revised: 12.5 .2020

Accepted: 14.5.2020 